\title{
Spatial and temporal visual filtering in patients with glaucoma and ocular hypertension
}

Abstract

Purpose To investigate the spatial and temporal response characteristics in patients with glaucoma and ocular hypertension (OHT), in order to demonstrate the earliest specific measure of vision loss and to elucidate the precise mechanism of early glaucomatous damage.

Methods The Open Optical View System was used to assess changes in spatial and temporal vision in patients with clearly defined glaucoma (30 eyes), patients with OHT (30 eyes) and 30 eye-matched normal control eyes. The psychophysical methods applied were developed by Barber and Ruddock, who showed that the responses called ST1 and ST2 have similar characteristics to those found in the parvocellular and magnocellular pathway respectively. The measurement of spatial and temporal responses rests upon the measurement of a target that moves across a background, itself modulated spatially (gratings) and temporally (flicker).

Results The results reported, taken together with the current data on retinal ganglion cell function, reveal damage to both the parvocellular and magnocellular pathway in patients with glaucoma. In the OHT groups only marginal changes were found in the parvocellular response, but significant changes in the magnocellular response. In both OHT and glaucoma, the temporal processing shows greatest abnormalities at a small number of low flicker frequencies. Conclusion We provide psychophysical evidence of the detectable ganglion cell damage in patients with OHT who remain normal on testing with automated perimetry. The psychophysical method chosen (which measures the function of specific sub-divisions of the visual pathway) may prove useful in screening for glaucomatous damage.

Key words Glaucoma, Ocular hypertension, Psychophysics, Spatial and temporal responses
Primary open angle glaucoma is a widespread human ocular disorder with optic nerve and retinal ganglion cell degeneration leading to blindness. In Britain it affects $1-2 \%$ of people over 40 years of age ( $3 \%$ of people over 50 years) and accounts for about 1 in 8 of new blind registrations. ${ }^{1}$ Recent analysis concluded that glaucoma is the second most frequent cause of blindness in the world after cataract. ${ }^{2}$ Three population-based surveys using regular screening techniques found that about $4 \%$ of white persons and $8 \%$ of black persons with glaucoma are legally blind (VA $\leqslant 20 / 200$ in both eyes). ${ }^{3}$ The mechanism of early glaucomatous visual loss is not fully understood. In patients functional consequences of glaucomatous damage are only readily observed at a relatively advanced stage, when both parvocellular and magnocellular pathways may be involved. If visual loss could be detected earlier, prophylactic therapy may prevent further functional loss.

A recent focus has been to isolate magnocellular function, ${ }^{4}$ since there is some histological evidence from both clinical and experimental data that larger ganglion cells are preferentially damaged in early glaucoma. 5,6 This group of cells projects to the magnocellular layers of the lateral geniculate nucleus, has a fast conduction velocity and is especially sensitive to transient change in retinal stimulation compared with the spectrally opponent parvocellular ganglion cells, which carry finer spatial information. There have been a number of psychophysical studies that have attempted to determine visual loss with magnocellular specificity, but the results remain equivocal. $^{7-9}$ Visual response abnormalities in glaucoma patients have been reported for spatial contrast sensitivity, ${ }^{10,11}$ transient response functions ${ }^{12-14}$ and colour discrimination. ${ }^{15,16}$ Evidence therefore exists that implicates the mechanisms mediated by the magnocellular pathway (transient responses), but also those mediated by the parvocellular pathway in the case of colour vision. The extent of these various visual dysfunctions has been
U. Vogt

C. Migdal

Western Eye Hospital

Marylebone Road

London NW1 5YE, UK

A Morland

K. Ruddock

Imperial College

London, UK

The authors would like to thank the Joint Standing Research Committee for their financial support 
correlated in some cases with other measures of visual disturbance, such as the severity of visual field scotomas and changes in the pattern electroretinogram (PERG) ${ }^{17}$ Thus, although the magnocellular pathway is thought to be damaged by the primary action of glaucoma, this has rarely been established.

In this paper we present the results of a psychophysical study in which we assessed a response function mediated by the magnocellular pathway, and another mediated by the parvocellular pathway. Patients with glaucoma and ocular hypertension $(\mathrm{OHT})$ were tested and compared with age-matched normal controls. Patients with OHT were tested because such patients can develop glaucoma and, therefore, may suffer damage consistent with early stages in the glaucoma disease process.

The psychophysical methods applied in this study were developed by Barber and Ruddock ${ }^{18}$ and by Holliday and Ruddock, ${ }^{19}$ who showed that the responses, called ST1 and ST2, have similar spatiotemporal characteristics to those found in the parvocellular and magnocellular pathways respectively. These methods had previously demonstrated sensitivity losses restricted to the ST1 response (the parvocellular pathway) in conditions such as amblyopia, albinism and residual visual function associated with hemianopia. ${ }^{20}$

\section{Subjects and methods}

In all the experiments reported here we determined the luminance of a moving target at which it could just no longer be detected by the subject. This value of target luminance is defined as the threshold target luminance, $I_{\mathrm{t}}$. In our experiments, $I_{\mathrm{t}}$ is measured for a target moving across a background field that was modulated either in time (i.e. flickered) or spatially, in which case it appeared as a grating. Although the average luminance of the background is maintained at a constant level, $I_{t}$ varies markedly with the frequency of the background modulation, and a plot of $I_{\mathrm{t}}$ against the background modulation frequency yields a frequency response curve. For normal observers, a plot of $\log I_{\mathrm{t}}$ against the spatial periodicity of the background grating peaks at 3-4 cycles per degree, falling on either side to give a well-defined band-pass response. This response 'ST1 spatial' was attributed to the spatial tuning of a visual mechanism with sustained temporal characteristics. ${ }^{19}$

Similarly, $I_{\mathrm{t}}$ for detection of a target moving across a flickering background peaks at a frequency of about $20 \mathrm{~Hz}$, to give a well-defined band-pass response, which Holliday and Ruddock ${ }^{19}$ named the 'ST2 temporal' response. In this case, the frequency at which $I_{t}$ is maximal varies with the average luminance of the background, which is maintained at a fixed value for all subjects

The light stimuli required for these measurements were generated by a purpose-built optical system, incorporating two commercial projectors, which provided two independent optical channels, one for the target and the other for the background. The images of the target and background components of the visual stimulus were projected onto a white screen. The circular target was imaged from a precision-drilled metal aperture, which was driven by a servo motor across the object plane of the 'target' projector to provide the target movement. The target luminance was controlled by two Polaroid filters, one of which was fixed whilst the other could be rotated. Spatial modulation of the background was achieved by placing a photographically produced, high-contrast (>95\%) grating in the object plane of the 'background projector', and the required periodicity values were selected from a set of gratings. The gratings were square-waveform in transmission profile, and their periodicities defined in cycles per degree. The luminance of the background field was controlled with neutral density filters and considerable care was taken in the photographic production of the gratings to produce a set for which each had the same optical density, which ensured that the background luminance was constant.

Temporal modulation of the flickering background was achieved by a rotating sector disc, placed in a defocused position beyond the projector lens, and driven by a variable frequency motor. This arrangement provided pseudo-sinusoidal modulation with a high modulation depth (>90\%), and relatively low harmonic distortion in the temporal frequency profile $(<10 \%)$.

In all these experiments the circular target was $3^{\circ}$ in diameter and moved horizontally at $15^{\circ}$ per second across the central $8^{\circ}$ of background grating. The circular background was $17^{\circ}$ in diameter, and of average luminance $1.8 \log$ troland.

\section{Protocol}

The subject was seated in front of a white screen onto which two standard slide projectors constituting two independent optical channels were projecting a background and a target respectively. The method of stimulus presentation was the setting of a target luminance using the Polaroid filters, the pressing of the control box button to begin the presentation enquiry of the subject as to whether the target had been detected and the setting of the new luminance on the basis of that answer. The target was set at a series of luminances around the threshold level, so as to simulate a staircase measurement of threshold luminance. ${ }^{21}$ A value of $I_{t}$ was only recorded if at least three successive turning points (changes in subject response from 'seen' to 'not seen' or vice versa) were made within a luminance range of $\pm 0.04 \log$ units. The luminance range of $\pm 0.04 \log$ units also provides the upper limit of the experimental error, although in practice errors were often smaller. The luminances of all stimuli were measured, in situ, with a Macam spectrophotometer/radiometer.

\section{Patients}

All patients taking part in this study were under the care of the Western Eye Hospital. They were examined ophthalmologically, including Snellen visual acuity, 


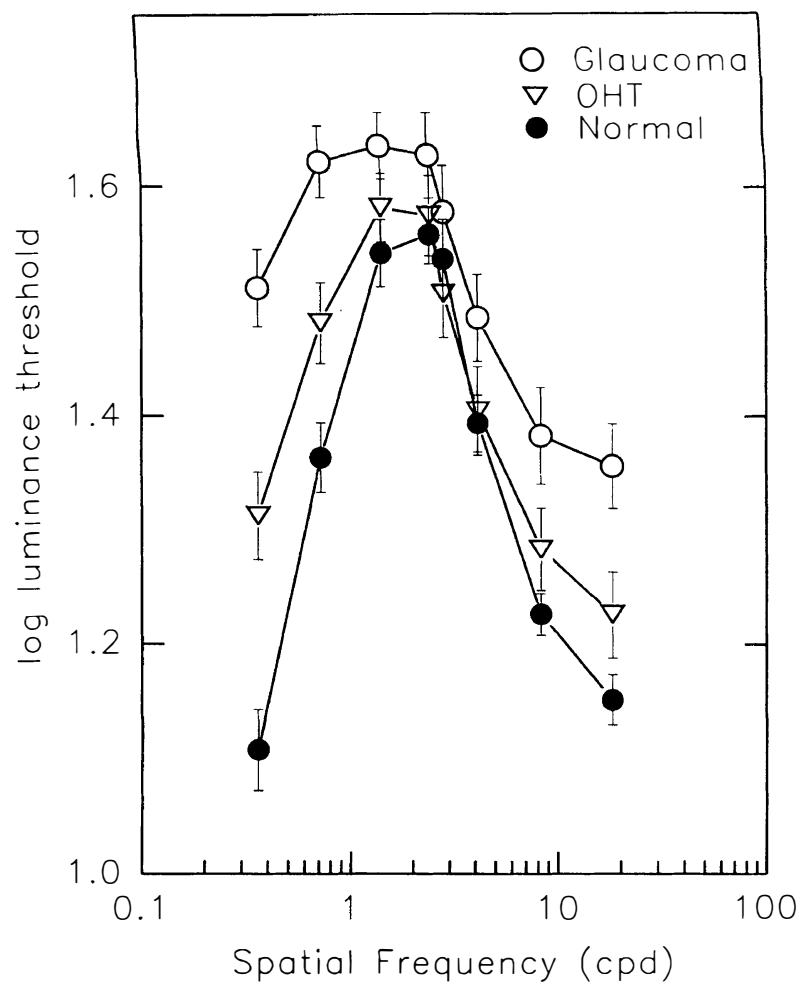

Fig. 1. The ST1 spatial response function: mean data in normals, ocular hypertensives (OHT) and glaucoma patients.

Goldmann applanation tonometry, fundoscopy and Humphrey visual field. Inclusion criteria were as follows:

1. Visual acuity (unaided or corrected with spectacles or contact lenses) $\geqslant 6 / 9$.

2. The 15 patients with bilateral OHT had normal Glaucoma Hemifield Test and Mean Deviation and Corrected Pattern Standard Deviation with normal limits (using the criterion of no points of the central 24-2 programme depressed by greater than $5 \mathrm{~dB}$ ) on the Humphrey field analyser. The cup/disc ratio in this group was 0.5 or less. All OHT patients had intraocular pressures (IOP) of $24 \mathrm{mmHg}$ or higher on at least three occasions. Seven of the patients in this group used Betagan (Allergan) on a regular basis. The patient group with primary open angle glaucoma had significant glaucomatous field loss and a cup/disc ratio between 0.5 and 0.95 . Two of the 16 patients in this group had a visual acuity of counting fingers in their other eye due to glaucoma. At the time of testing all patients in this group had controlled IOPs on treatment with topical beta-blockers.
The normal control group had normal visual acuity, fields that showed no evidence of glaucomatous loss and IOPs within normal limits (between 11 and $18 \mathrm{mmHg}$ ) with cup/disc ratios of between 0.1 and 0.4 .

The age of the 16 glaucoma patients (30 eyes) ranged from 54 to 89 years (mean 71.5 years \pm 9 years), that of the 15 OHT patients from 49 to 80 years (mean 65.4 years \pm 9 years) and that of the normal control group (15 patients = 30 eyes) from 45 to 85 years (mean 66.5 years \pm 10 years). The age differences between the groups are statistically insignificant.

This study was approved by the ethics committee of the Western Eye Hospital and was undertaken with the understanding and consent of each subject.

\section{Results}

\section{The ST1 spatial response function}

The values of $\log I_{\mathrm{t}}$ are plotted as a function of background grating spatial frequency in Fig. 1. The response functions defined by these plots show clear differences between the three observer groups. The averaged data of the normal observers displays the usual band-pass response with a peak threshold value at 2.82 cycles per degree (c/deg), characteristics which have been previously reported by Morland et al. ${ }^{22}$

The response derived from averaged data of the patient group with OHT has a similar form to that of the normal response, but the peak threshold value occurs at the lower spatial frequency of $1.41 \mathrm{c} / \mathrm{deg}$. In addition, threshold values are elevated, compared with those of the normal response, below $1.2 \mathrm{c} / \mathrm{deg}(p<0.001)$.

Thresholds obtained at the two highest frequencies, 8.34 and $18.18 \mathrm{c} / \mathrm{deg}$, were also significantly higher $(p=0.01)$ for the OHT group than the normal control group.

The threshold values measured in the glaucoma patient group show a large difference from those of the normals. The peak threshold is at $1.41 \mathrm{c} / \mathrm{deg}$ and is significantly elevated $(p=0.028)$ compared with the normal value. At spatial frequencies of 0.72 and 0.36 , the threshold values for the glaucoma group are elevated above those of the normals by approximately 0.3 and 0.4 $\log$ units respectively. The differences observed at these low spatial frequency values are highly significant $\left(p<1 \times 10^{-5}\right.$ and $p<1 \times 10^{-10}$ for 0.72 and $\left.0.36 \mathrm{c} / \mathrm{deg}\right)$. At spatial frequencies higher than the response peak, thresholds are elevated compared with those of the normal group, with $p=0.046,0.0016$ and $2 \times 10^{-5}$ for $4.12,8.24$ and $18.18 \mathrm{c} / \mathrm{deg}$ respectively (Table 1 ).

Table 1. Statistical significance of differences between groups in the ST1 spatial response function

\begin{tabular}{lccccccccc}
\hline & \multicolumn{5}{c}{ Spatial frequency (c/deg) } \\
\cline { 2 - 8 } Comparison & 0.36 & 0.72 & 1.41 & 2.14 & 2.82 & 4.12 & 8.24 \\
\hline Glaucoma vs normals $^{\mathrm{a}}$ & $\mathbf{2 E - 1 1}$ & $\mathbf{1 E - 0 6}$ & $\mathbf{0 . 0 2 8 1}$ & 0.1304 & 0.4459 & 0.0468 & $\mathbf{0 . 0 0 1 6}$ & $\mathbf{2 E - 0 5}$ \\
OHT vs normals & $\mathbf{8 E - 0 5}$ & $\mathbf{0 . 0 0 1}$ & 0.3065 & 0.3903 & 0.3222 & 0.2011 & 0.0518 & $\mathbf{0 . 0 2 4 7}$ & 0.18 \\
Glaucoma vs OHT & $\mathbf{2 E - 0 5}$ & $\mathbf{0 . 0 1 0 1}$ & 0.169 & 0.1635 & 0.7192 & 0.0544 & $\mathbf{0 . 0 1 0 2}$ & $\mathbf{0 . 0 1 1 7}$ \\
\hline
\end{tabular}

OHT, ocular hypertension.

${ }^{a}$ Unpaired two-tailed $t$-test of unequal variance. 


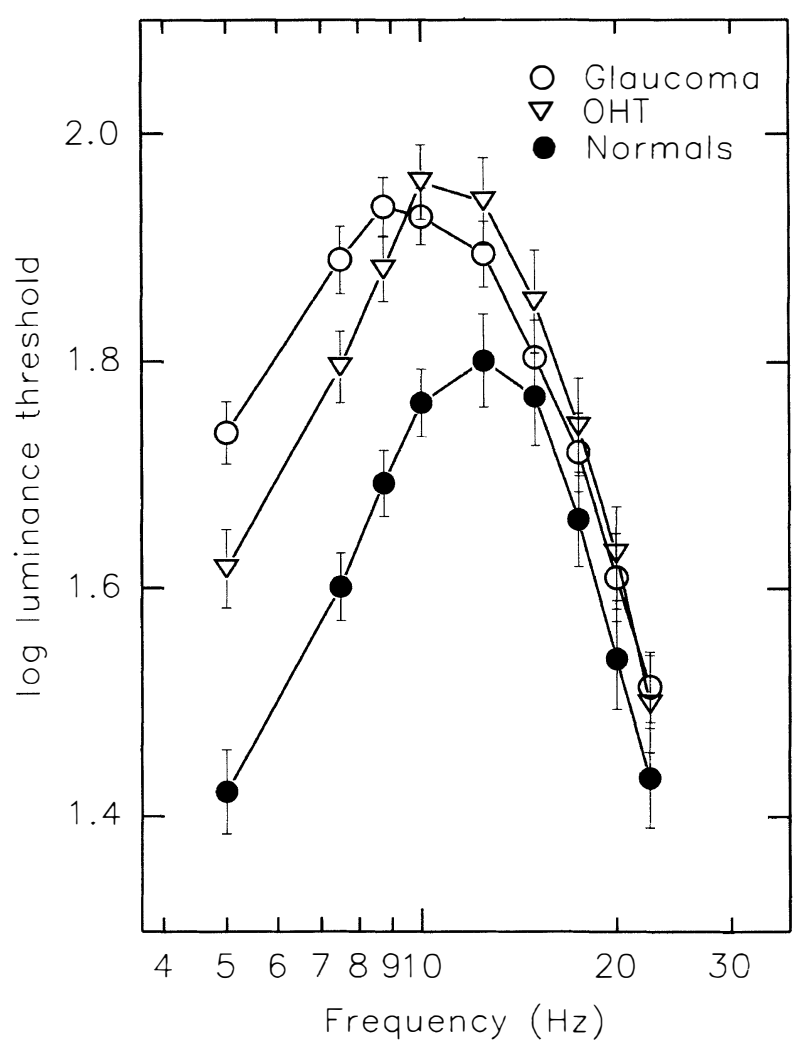

Fig. 2. The ST2 temporal response function: mean data in normals, ocular hypertensives (OHT) and glaucoma patients.

In summary, the change in the ST1 spatial response function for both patients with OHT and those with glaucoma is to shift the response to lower spatial frequency and to elevate thresholds in general. The effect is considerably more severe in the patients with glaucoma than those with OHT. Moreover, when the two patients groups are compared, highly significant differences $(p<0.02)$ are observed at $0.36,0.72,8.24$ and $18.18 \mathrm{c} / \mathrm{deg}$ (Table 1).

\section{The ST2 temporal response function}

Data for the ST2 temporal responses are presented as log $I_{\mathrm{t}}$ values plotted as a function of the background flicker frequency (Fig. 2). This data plot yields the temporal response for the three observer groups, and as with the spatial response measured, clear differences between groups are shown. The response function of the normal control group displays the band-pass characteristics shown in previous evaluations of the mechanism. ${ }^{19,20,22}$ The peak threshold value is obtained at $12.5 \mathrm{~Hz}$, which is consistent with the data previously collected at the average background luminance of $1.4 \mathrm{log}$ troland employed in this study.
The averaged data of the OHT group gives rise to a response function that is markedly different from that of the normals. The peak threshold value for the OHT patient group is obtained at $10 \mathrm{~Hz}$, and all values of threshold below $12.5 \mathrm{~Hz}$ are significantly elevated above those of the normals $(p<0.001)$ (Table 2). At flicker frequencies above and equal to $12.5 \mathrm{~Hz}$, the response functions are not significantly different $(p>0.1)$.

The response function derived from averaging data of the glaucoma patients displays a deviation from the normal response similar to that of the OHT response. The peak threshold is obtained at $8.75 \mathrm{~Hz}$ and all threshold values obtained below flicker frequencies of $12.5 \mathrm{~Hz}$ are significantly elevated $(p<0.0001)$. At flicker frequencies at $12.5 \mathrm{~Hz}$ and greater, the threshold values of the glaucoma patient group show little deviation from those of the normals $(p>0.07)$.

In summary, the ST2 temporal responses show variation between groups at flicker frequencies below $12.5 \mathrm{~Hz}$. The ST2 temporal response is modified more in glaucoma than in OHT compared with the normal response. When the two patient groups are compared, a highly significant $(p<0.02)$ difference is only observed at $5 \mathrm{~Hz}$. However, when the OHT group is compared with the normals, highly significant differences are revealed in flicker frequencies from 5, 7.5, 8.75, 10 and $12.5 \mathrm{~Hz}$ (Table 2).

\section{Discussion}

There is considerable interest in the early detection of glaucoma. Long-term studies have shown that eyes with long-standing OHT can subsequently develop primary open angle glaucoma with an incidence of about $1 \%$ per year: after 5 years $5 \%$ and after 10 years in the order of $10 \% .{ }^{23}$ Methods for screening glaucoma and monitoring OHT patients depend upon testing the integrity of different parts of the visual pathway. Much evidence has been accumulated from psychophysical testing, ${ }^{24-28}$ electrophysical testing, ${ }^{29-31}$ histological examination ${ }^{5,6,32}$ and clinical evaluation of the optic disc. ${ }^{33-36}$ Quigley $^{2}$ stated that although there was loss of all cell types, larger ganglion cells appeared to be more affected. Johnson's ${ }^{37}$ psychophysical data revealed an overall amount of loss for all nerve fibres irrespective of their diameter, and recent papers by Graham et al. ${ }^{8}$ and Sample et al. ${ }^{38,39}$ found no preferential loss of large ganglion cells in psychophysical testing.

The different visual cortical pathways have their origin in two different types of retinal ganglion cells. The P-type terminates in the P layers of the lateral geniculate nucleus (LGN) and has general characteristics suitable

Table 2. Statistical significance of differences between groups in the ST2 temporal response function

\begin{tabular}{lcccccccccc}
\hline & \multicolumn{3}{c}{ Flicker frequency $(\mathrm{Hz})$} \\
\cline { 2 - 8 } Comparison & 5 & 7.5 & 8.75 & 10 & 12.5 & 15 & 17.5 & 20 \\
\hline Glaucoma vs normals & $\mathbf{8 E - 0 9}$ & $\mathbf{5 . 6 E - 0 9}$ & $\mathbf{5 E - 0 8}$ & $\mathbf{0 . 0 0 0 1 1}$ & 0.06973 & 0.53012 & 0.28016 & 0.22521 & 0.14407 \\
OHT vs normals & $\mathbf{0 . 0 0 0 3}$ & $\mathbf{3 . 8 9 E - 0 5}$ & $\mathbf{2 E - 0 5}$ & $\mathbf{5 . 4 E - 0 5}$ & $\mathbf{0 . 0 1 6 3 1}$ & 0.18731 & 0.17841 & 0.12875 & 0.29289 \\
Glaucoma vs OHT & $\mathbf{0 . 0 0 9 1}$ & $\mathbf{0 . 0 3 3 8 2}$ & 0.15659 & 0.46525 & 0.34251 & 0.38341 & 0.68445 & 0.71264 & 0.77803 \\
\hline
\end{tabular}


for form and colour vision, while the M-type terminates in the $M$ layers and has characteristics that make it suitable for detecting dynamic form and motion. Different signals from the retina are relayed to area V1 through the P and M layers of the LGN. The cortical termination sites (in V1) derived from the two sets of LGN layers themselves have very specific and different visual cortical areas. It has been suggested that the entire visual pathways in the cerebral cortex can be referred to as the $\mathrm{P}$ or the $\mathrm{M}$ pathway. ${ }^{40}$

Because of the segregation of visual pathways we are able to isolate specific functions using psychophysical techniques. The low-pass ST1 spatial and band-pass ST2 temporal responses respectively define channels with sustained and transient characteristics. They therefore correspond to the two principal classes of electrophysiological pathway. The larger receptive fields associated with the transient ST2 channel are also consistent with the properties of the electrophysical units.

As explained previously the sustained and transient units loosely correspond to the X-and Y-cell classification respectively. Thus ST1 and ST2 channels can be regarded as psychophysical correlates of $\mathrm{X}$ - and Y-type (magno- and parvocellular). However, there is ample opportunity for the $\mathrm{P}$ and $\mathrm{M}$ signal to mix in the cortex, ${ }^{41-43}$ so that the input of the specialised visual areas may consist of signals from either source.

If damage is occurring in both the magnocellular and parvocellular divisions of the visual pathway, the question remains as to whether there is a diffuse loss of ganglion cells or whether the results are due to pathway interaction.

We report abnormalities in both the ST1 spatial and ST2 temporal visual responses of patients with OHT and glaucoma. The abnormalities in the ST1 spatial response correspond to damage to the parvocellular pathway. The parvocellular pathway is also of higher resolution than the magnocellular pathway and therefore limits visual acuity, which was worst in the glaucoma patients at $0.86 \pm 0.04$; the OHT patients had similar acuity $(1.01 \pm 0.02)$ to the controls $(1.07 \pm 0.03)$. The changes in the ST1 spatial response are very clear in the glaucoma patient group, the average response of which is tuned to lower spatial frequencies. The data for the OHT patients show a far greater similarity to the normal response curve (Fig. 1), but there are statistically significant differences between the two responses at low spatial frequencies. It should be noted, however, that the differences between the responses of the OHT and glaucoma patients and that of normals are not as severe as those between amblyopes and controls. ${ }^{44}$ The abnormalities in the ST1 responses of patients with glaucoma are consistent with other psychophysical tests that reveal parvocellular dysfunction in glaucoma. ${ }^{15,16,28}$ In addition, measures of contrast sensitivity also showed low spatial frequency abnormalities in patients with glaucoma, ${ }^{10}$ consistent with our results.
The ST2 temporal response function is also disrupted in patients with OHT and glaucoma, and as with the ST1 spatial response the more severe change in the response is observed in the glaucoma patients compared with the OHT group. The test applied is not dependent on the visual acuity of the observer and reflects the action of magnocellular units. ${ }^{19}$ The results demonstrate, therefore, that both patients with OHT and those with glaucoma suffer damage to the magnocellular pathways. Of particular interest is the large difference in the response of the OHT patients and the normal controls, which is larger than that found in the ST1 spatial response. In the patients with glaucoma who we tested, damage to both the parvocellular and the magnocellular pathways is implicated, whereas in the OHT group only marginal changes were found in the parvocellular response, compared with gross changes in the magnocellular response. Despite the similarity in the ST1 and ST2 graphs the importance lies in the statistical $p$ values, which make the OHT ST2 results much more significant. Studies on motion perception, which is mediated by the magnocellular pathway, have shown abnormal responses in glaucoma. ${ }^{13}$ Measures of flicker sensitivity have also revealed abnormal processing in the magnocellular pathway in glaucoma. ${ }^{14}$ Our results are, therefore, consistent with previous studies.

The significance of this study's findings is that the OHT data demonstrate a change in psychophysical responses consistent with damage to the magnocellular cells. The losses in OHT patients are statistically much more significant with the ST2 temporal response only (Table 2). We provide psychophysical evidence, therefore, of the presence of significant ganglion cell damage in OHT patients despite the fact that their automated perimetry tests are normal.

In addition, we have shown that in both OHT and glaucoma the temporal processing produces greatest abnormalities at low flicker frequencies. This test could, therefore, be applied at a small number of low flicker frequencies, where threshold differences are greatest, in order to screen a large number of patients with OHT and glaucoma.

The conclusion can be drawn that the ST1 and ST2 channel response characteristics in glaucoma and OHT are coarser than in normal vision, suggesting damage to both the magnocellular and parvocellular pathways. Both large- and small-diameter ganglion cells are affected early in the disease, but our measurements on patients with OHT indicate that it is to a lesser degree than glaucoma. The results suggest that the extent of the damage in the magnocellular pathway exceeds parvocellular damage in the OHT group. We have shown that the ganglion cell damage precedes the field defect in the OHT cases. The psychophysical method applied in this study, therefore, appears to be of use in detecting early glaucomatous damage in the OHT group. A prospective long-term follow-up study is required to demonstrate which individuals with abnormal responses will eventually develop glaucomatous field loss. 


\section{References}

1. Jay JL, Murdoch JR. The rate of visual field loss in untreated primary open angle glaucoma. $\mathrm{Br} \mathrm{J}$ Ophthalmol 1993;77:176-8

2. Quigley HA. The number of persons with glaucoma worldwide. Br J Ophthalmol 1996;80:389-93.

3. Quigley HA, Vitale S. Models of glaucoma prevalence and incidence in the United States. Invest Ophthalmol Vis Sci 1997;38:83-91.

4. Johnson CA, Samuels SJ. Screening for glaucomatous visual field loss with frequency doubling perimetry. Invest Ophthalmol Vis Sci 1997;38:413-25.

5. Quigley HA, Sanchez RM, et al. Chronic glaucoma selectivity damages large optic nerve fibres. Invest Ophthalmol Vis Sci 1987:28:913-20.

6. Quigley HA, Dunkelberger GR, Green WR. Chronic human glaucoma causing selectively greater loss of large optic nerve fibres. Ophthalmology 1988;95:357-64.

7. Bosworth CF, Sample PA, Weinreb RN. Motion perception thresholds in the areas of glaucomatous field loss. Vision Res 1997;37:355-64.

8. Graham SL, Drance SM, Chauham BC, et al. Comparison of psychophysical and electrophysical testing in early glaucoma. Invest Ophthalmol Vis Sci 1997;37:2651-2.

9. Bosworth CF, Sample PA, Weinreb RN. Perimetric motion thresholds are elevated in glaucoma suspects and glaucoma patients. Vision Res 1997;37:1989-97.

10. Arden GB, Jacobson JJ. A simple grating test for contrast sensitivity: preliminary results in screening for glaucoma. Invest Ophthalmol Vis Sci 1978;17:23-32.

11. Sokol S, Domar A, Moskowitz A, Schwartz B. Pattern provoked potential latency and contrast sensitivity in glaucoma and ocular hypertension. Doc Ophthalmol 1981;27:79-86.

12. Fitzke FW, Poinoosawmy D, Nagasubramanian S, Hitchings RA. Peripheral displacement thresholds in glaucoma and ocular hypertension. Perimetry update 1988. VIIIth International Perimetric Society meeting. Amsterdam: Kugler \& Ghedini, 1989:399-405.

13. Wu J, Fitzke F, Poinoosawmy D, Hitching R. Four years follow up of the fellow eye of unilateral glaucoma patients using motion sensitivity. Invest Ophthalmol Vis Sci 1994;35:2185.

14. Tyler CW. Specific deficits of flicker sensitivity in glaucoma and ocular hypertension. Invest Ophthalmol Vis Sci 1981;20:204-12.

15. Adams AJ, Heron G, Husted R. Clinical measures of central vision function in glaucoma and ocular hypertension. Arch Ophthalmol 1987;105:782-7.

16. Falcao-Reis FM, Spileers W, O'Sullivan F, Arden GB, et al. Macular colour contrast sensitivity in ocular hypertension and glaucoma. Br J Ophthalmol 1991;75:598-602.

17. Sieving PA, Linsenmeir RA, Steinberg RH. Current source density (CSD) analysis shows main pattern ERG (PERG) source in proximal retina. Invest Ophthalmol Vis Sci 1984;25(Suppl):259.

18. Barber JL, Ruddock KH. Spatial characteristics of movement detection mechanisms in human vision: achromatic mechanisms. Biol Cybern 1980;37:72-92.

19. Holliday IE, Ruddock KH. Two spatio-temporal filters in human vision: temporal and spatial frequency response characteristics. Biol Cybern 1983;47:137-40.

20. Bromley IM, Ruddock KH. Visual spatial filtering and pattern discrimination are normal in strabismic amblyopia. Clin Vision Sci 1987;1:209-18.

21. Cornswet TN. The staircase method in psychophysics. Am J Psychol 1962;75:485-91.

22. Morland $\mathrm{AB}$, Bronstein $\mathrm{AM}$, Ruddock $\mathrm{KH}$. Vision during motion in patients with absent vestibular function. Acta Otolaryngol (Stockh) 1995;(Suppl 520):338-42.
23. David R, Livingstone D, Lunz MH. Ocular hypertension: a long term follow up of treated and untreated patients. $\mathrm{Br} \mathrm{J}$ Ophthalmol 1997;61:663-74.

24. Gunduz K, Arden GB, Perry S, Weinstein G, Hitchings RA Colour vision defects in ocular hypertension and glaucoma: quantification with a computer driven colour television system. Arch Ophthalmol 1988;108:929-35.

25. Joffe KM, Raymond JE, Crichton A. Motion perimetry in glaucoma. Invest Ophthalmol Vis Sci 1991,32:1103.

26. Drance SM, Lakowski R, Schulzer M, Douglas GR. Acquired colour vision changes in glaucoma. Arch Ophthalmol 1981;99:829-31.

27. Johnson CA, Adas AJ, Casson EJ, Quigg JM. Can short wavelength sensitivity losses predict the development of glaucomatous visual field defects? Invest Ophthalmol Vis Sci 1991;32:1191.

28. Yu TC, Falcao-Reis FM, Spileers W, Arden GB. Peripheral colour contrast: a new screening test for pre-glaucomatous vision loss. Invest Ophthalmol Vis Sci 1991;32:2279-89.

29. Maffei L, Fiorentini A. Electroretinographic responses to alternating gratings before and after section of the optic nerve. Science 1984;211:953-5.

30. May JG, Ralston JV, Reed JL, vanDyk HJL. Loss of patternelicited electroretinograms in optic nerve dysfunction. Am J Ophthalmol 1982;93:418-22.

31. Ringens PJ, Viifvinkel-Bruinenga S, Van Lith GHM. The pattern elicited electroretinogram. I. A tool in the early detection of glaucoma. Ophthalmologica 1986;192:171-5.

32. Quigley HA, Dunkelberger GR, Green WR. Retinal ganglion cell atrophy correlated with automated perimetry in human eyes with glaucoma. Am J Ophthalmol 1989;107:453-64.

33. Jonas JB, Gusek GC, Naumann GOH. Optic disc morphometry in chronic open angle glaucoma: morphometric intrapapillary characteristics. Graefes Arch Clin Exp Ophthalmol 1988;226:522-30.

34. Nicolela MT, Walman BE, Buckley AR, Drance SM. Various glaucomatous optic nerve appearances: a colour Doppler imaging study of retrobulbar circulation. Can J Ophthalmol 1996;103:1670-9.

35. Lester M, Mikelberg FS, Drance SM. The effect of optic disc size on diagnostic precision with the Heidelberg Retina Tomograph. Ophthalmology 1997;104:545-8.

36. Airakksinen PJ, Drance SM, Schulzer M. Neuroretinal rim area in early glaucoma. Am J Ophthalmol 1985;99:1-4.

37. Johnson CA. Selective versus non-selective losses in glaucoma. J Glaucoma 1994;3:32-44.

38. Sample PA, Irak I, Martinez GA, Yamagishi N. Asymmetries in the normal short wavelength visual field: implications for short wavelength automated perimetry. Am J Ophthalmol 1997;124:46-52.

39. Sample PA, Martinez GA, Weinreb RN. Short wavelength automated perimetry without lens density testing. Am J Ophthalmol 1995;118:632-41.

40. Livingstone MS, Hubel DH. Psychophysical evidence for separate channels for the perception of form, colour, movement and depth. J Neurosci 1987;7:3416-68.

41. Blasdel GG, Lund JS, Fitzpatrick D. Intrinsic connections of macaque striate cortex: axonal projections of cells outside lamina 4C. J Neurosci 1985;5:3350-69.

42. Rockland KS. A reticular pattern of intrinsic connections in primate area V2 (area 18). J Comp Neurol 1985;235:467-78.

43. Shipp S, Zeki S. The organisation of connections between areas V5 and V1 in macaque monkey visual cortex. Eur J Neurosci 1989;1:309-32.

44. Grounds AR, Holliday IE, Ruddock KH. I. Two spatiotemporal filters in human vision. II. Selective modification in amblyopia, albinism and hemianopia. Biol Cybern 1983;47:191-201. 